\title{
Dirac neutrino magnetic moment and a possible time evolution of the neutrino signal from a supernova
}

\author{
R. A. Anikin, A. V. Kuznetsov ${ }^{\dagger}$ N. V. Mikheev ${ }^{\ddagger}$ \\ Yaroslavl State P.G. Demidov University \\ Sovietskaya 14, 150000 Yaroslavl, Russian Federation
}

\begin{abstract}
We analyze the influence of neutrino helicity conversion, $\nu_{L} \rightarrow \nu_{R}$, on the neutrino flux from a supernova, caused by the interaction of the Dirac neutrino magnetic moment with a magnetic field. We show that if the neutrino has a magnetic moment in the interval $10^{-13} \mu_{\mathrm{B}}<\mu_{\nu}<10^{-12} \mu_{\mathrm{B}}$ and provided that a magnetic field of $\sim 10^{13}-10^{14} \mathrm{G}$ exists in the supernova envelope, a peculiar kind of time evolution of the neutrino signal from the supernova caused by the resonance transition $\nu_{L} \rightarrow \nu_{R}$ in the magnetic field of the envelope can appear. If a magnetar with a poloidal magnetic field is formed in a supernova explosion, then the neutrino signal could have a pulsating behavior, i.e., a kind of a neutrino pulsar could be observed, when it rotates around an axis that does not coincide with its magnetic moment and when the orientation of its rotation axis is favourable for our observation.
\end{abstract}

\section{Introduction}

The processes of helicity flip, $\nu_{L} \longleftrightarrow \nu_{R}$, are possible for a nonzero neutrino magnetic moment. In the case of a Dirac neutrino, their realization under conditions of a magnetized plasma in astrophysical objects can be an important factor for the mechanism of energy losses by such objects. In the standard model extended to include the neutrino mass $m_{\nu}$, the neutrino magnetic moment is known to be [1,2]

$$
\mu_{\nu}^{(S M)}=\frac{3 e G_{\mathrm{F}} m_{\nu}}{8 \pi^{2} \sqrt{2}}=3.20 \times 10^{-19}\left(\frac{m_{\nu}}{1 \mathrm{eV}}\right) \mu_{\mathrm{B}}
$$

where $\mu_{\mathrm{B}}=e / 2 m_{e}$ is the Bohr magneton 1 . Given the existing constraints on the neutrino masses, this quantity may be considered unobservably small. On the other hand, various nontrivial extensions of the standard model, such as the broken left-right symmetry [3 7], admit considerably larger neutrino magnetic moments [8-10].

Considerable interest in the neutrino magnetic moment arose after the momentous event of the SN 1987A explosion [11-16] in connection with the modeling a supernova explosion in which the huge outgoing neutrino flux essentially determines the energetics of the process. This means that such a microscopic neutrino characteristic as the magnetic moment could have a decisive effect on the macroscopic properties of these astrophysical events.

Two possible mechanisms for the realization of neutrino helicity flip $\nu_{L} \longleftrightarrow \nu_{R}$ in astrophysical conditions are discussed in the literature.

\footnotetext{
*e-mail: anik-roman@mail.ru

${ }^{\dagger}$ e-mail: avkuzn@uniyar.ac.ru

${ }^{\ddagger}$ e-mail: mikheev@uniyar.ac.ru

${ }^{1}$ We use a natural system of units with $c=\hbar=1$. $e>0$ is the elementary charge.
} 
(1) The scattering mechanism is caused by the interaction of the Dirac neutrino magnetic moment with the microscopic electromagnetic field of a virtual plasmon. For example, interacting with the plasmon that can be both produced and absorbed, the trapped left-handed neutrinos in a supernova core can be converted into right-handed ones:

$$
\nu_{L} \rightarrow \nu_{R}+\gamma^{*}, \quad \nu_{L}+\gamma^{*} \rightarrow \nu_{R}
$$

These right-handed neutrinos are sterile with respect to weak interactions, which can be important, for example, when the energy losses by stars are taken into account. The overly large flux of right-handed neutrinos produced in such interactions from the supernova core may not leave a sufficient amount of energy to explain the observed supernova neutrino luminosity. Thus, an upper bound on the neutrino magnetic moment can be established (see, e.g., [17-19]; for a more detailed list, see [20]). The contribution from the neutrino helicity flip process to the supernova core luminosity was investigated most consistently in our recent paper [21]. Here, instead of the model of a homogeneous sphere used in previous studies, we considered realistic models with radial distributions and time evolution of physical parameters in the supernova core. We obtained upper limits on the flavor-averaged Dirac neutrino magnetic moment from the condition that the influence of right-handed neutrino emission on the total cooling timescale should be limited,

$$
\bar{\mu}_{\nu}<(1.1-2.7) \times 10^{-12} \mu_{\mathrm{B}},
$$

depending on the explosion model.

(2) The mechanism of oscillations $\nu_{L} \leftrightarrow \nu_{R}$ can be realized when the neutrino magnetic moment interacts with a macroscopic magnetic field in a supernova envelope. The outgoing flux of right-handed neutrinos from the core during collapse falls into the region of the supernova envelope between the neutrinosphere (of radius $R_{\nu}$ ) and the shock stagnation zone (of radius $R_{s}$ ). According to existing views, typical values of these quantities change insignificantly in the stagnation time and can be estimated as $R_{\nu} \sim 20-50 \mathrm{~km}$ and $R_{s} \sim 100-200 \mathrm{~km}$. If a sufficiently strong magnetic field, of the order of its critical value of $B_{e}=m_{e}^{2} / e \simeq 4.41 \times 10^{13} \mathrm{G}$, is present in the region under consideration, then neutrino spin oscillations take place.

The interesting possibility of a combination of both these mechanisms was first suggested by Dar [22], who considered the process of double neutrino helicity conversion, $\nu_{L} \rightarrow \nu_{R} \rightarrow \nu_{L}$, under supernova conditions, where the first stage is realized through the interaction of the neutrino magnetic moment with plasma electrons and protons in the supernova core and the second stage arises from the neutrino spin flip in the magnetic field of the envelope. Voloshin [23] additionally took into account the possibility of resonant conversion of right-handed neutrinos into left-handed ones, $\nu_{R} \rightarrow \nu_{L}$, that was not considered by Dar [22]. In our recent paper [24], we reanimated the idea of Dar [22] based on a new refined estimate [20] for the flux and luminosity of right-handed neutrinos from the central part of a supernova (the previously used data were underestimated significantly). We determined the conditions under which the mechanism of double neutrino helicity conversion could stimulate a damped shock during a supernova explosion.

Here, we discuss a possibility of a combined action of a magnetic field and medium in the SN envelope on the outgoing neutrinos which could cause the resonant transition $\nu_{L} \rightarrow \nu_{R}$, and thus the SN neutrino signal could be modified. In principle, this effect could be observable. This talk is based on our recent paper [25].

\section{Neutrino helicity flip in a weakly magnetized plasma}

It is most convenient to illustrate the influence of a magnetic field on a neutrino with a magnetic moment using the equation for neutrino helicity evolution in an external magnetic field. 
Given the additional energy acquired by the left-handed electron neutrinos $\nu_{e}$ in the supernova envelope, the helicity evolution equation can be written as [23,26-31]

$$
\mathrm{i} \frac{\partial}{\partial t}\left(\begin{array}{c}
\nu_{R} \\
\nu_{L}
\end{array}\right)=\left[\hat{E}_{0}+\left(\begin{array}{cc}
0 & \mu_{\nu} B_{\perp} \\
\mu_{\nu} B_{\perp} & C_{L}
\end{array}\right)\right]\left(\begin{array}{c}
\nu_{R} \\
\nu_{L}
\end{array}\right)
$$

where

$$
C_{L}=\frac{3 G_{\mathrm{F}}}{\sqrt{2}} \frac{\rho}{m_{N}}\left(Y_{e}-\frac{1}{3}\right) .
$$

Here, the ratio $\rho / m_{N}=n_{B}$ is the nucleon number density, $Y_{e}=n_{e} / n_{B}=n_{p} / n_{B}, n_{e, p}$ are the electron and proton number densities respectively, and $B_{\perp}$ is the transverse magnetic field component with respect to the direction of neutrino motion; the term $\hat{E}_{0}$ is proportional to a unit matrix and is unimportant for our analysis.

It should be explained why we use Eq. (5) for the additional energy of the left-handed electron neutrinos in an unpolarized medium, although, in general, at least a partial polarization of electrons should arise in a field of the order of $B_{e}$. In this case, the validity of the unpolarizedmedium approximation is seen from the following considerations. As is well known, the states of electrons in a magnetic field corresponding to all Landau levels, except the ground level, are doubly degenerate with respect to the spin projection onto the field direction and, thus, do not contribute to the polarization of the medium. Therefore, to estimate the degree of polarization, it will suffice to estimate the fraction of electrons populating the ground Landau level whose spins are uncompensated. For typical conditions of the supernova envelope region under consideration, the electron chemical potential is $\tilde{\mu}_{e} \simeq 5-10 \mathrm{MeV}$ (see, e.g. [32]). Hence, dividing the number density of the electrons populating the ground Landau level, $n_{0} \simeq e B \tilde{\mu}_{e} /\left(2 \pi^{2}\right)$, by the total electron number density, $n_{e} \simeq \tilde{\mu}_{e}^{3} /\left(3 \pi^{2}\right)$, we obtain an estimate for the degree of polarization of the medium:

$$
P \sim \frac{n_{0}}{n_{e}} \lesssim \frac{e B}{\tilde{\mu}_{e}^{2}} \sim 10^{-2} \frac{B}{B_{e}} .
$$

Thus, for the magnetic field strengths $B \sim B_{e}$ under consideration, using the unpolarizedmedium approximation is justified. A more rigorous condition of weak plasma magnetization under which the influence of the magnetic field on the polarization of the medium can be neglected is formulated as

$$
B \ll \frac{\left(3 \pi^{2} n_{e}\right)^{2 / 3}}{e} \simeq 0.6 \times 10^{16} \mathrm{G}\left(\frac{n_{e}}{10^{33} \mathrm{~cm}^{-3}}\right)^{2 / 3} .
$$

Expression (5) for the additional energy of the left-handed neutrinos $C_{L}$ deserves a special analysis. A remarkable possibility is that this quantity becomes zero precisely in the supernova envelope region of interest to us [23. This, in turn, is a condition for the resonance transition $\nu_{R} \rightarrow \nu_{L}$ in the form $Y_{e}=1 / 3$. It should be noted that the values of $Y_{e}$ typical of collapsing matter, $Y_{e} \sim 0.4-0.5$, are realized in the supernova envelope. However, causing the dissociation of heavy nuclei, the shock wave makes the matter more transparent to neutrinos. This leads to the so-called "short" neutrino burst and, as a consequence, to considerable deleptonization of the matter in this region. According to existing views, a characteristic dip where $Y_{e}$ can drop to $\sim 0.1$ arise in the radial distribution of $Y_{e}$ (see, e.g. [32, 33]). Thus, there inevitably exists a point where $Y_{e}$ takes on a value of $1 / 3$. Remarkably, there is only one such point with $\mathrm{d} Y_{e} / \mathrm{d} r>0$, see $[32,33]$.

Note that $Y_{e}=1 / 3$ is a necessary but still insufficient condition for resonant conversion of right-handed neutrinos into left-handed ones, $\nu_{R} \rightarrow \nu_{L}$. The adiabaticity condition should also be met. It means that when shifted from the resonance point to a distance of the order of the oscillation length, the diagonal element $C_{L}$ in Eq. (4) at least should not exceed considerably the nondiagonal element $\mu_{\nu} B_{\perp}$. 


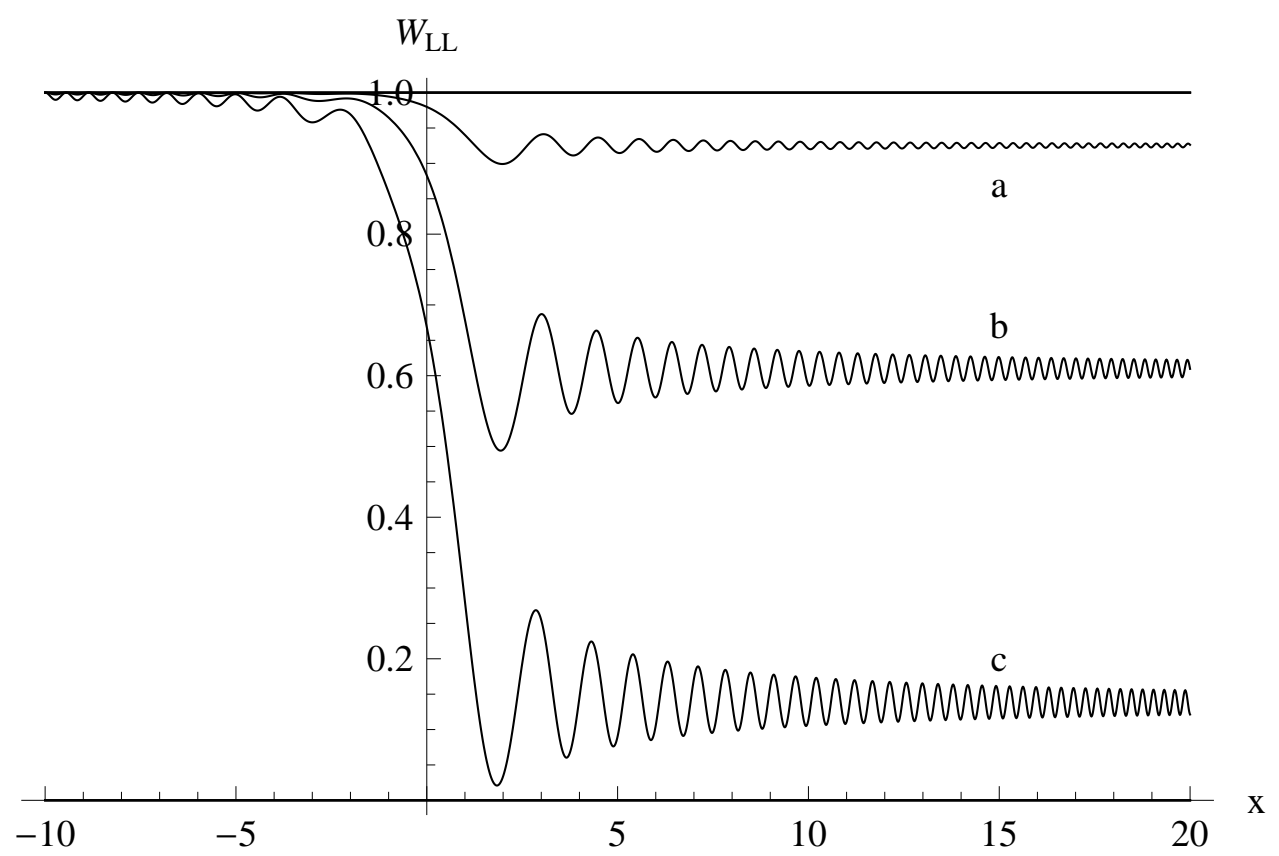

Figure 1: Pattern of variations in $W_{L L}$, the survival probability of left-handed neutrinos, $\nu_{e L} \rightarrow$ $\nu_{e L}$, (transparency), with distance $x$ (in arbitrary units) when passing through the resonance point placed at the coordinate origin for several magnetic field strengths: $B=0.2 B_{e}$ (a); $B=0.5 B_{e}$ (b); $B=B_{e}$ (c). To be specific, the neutrino magnetic moment is assumed to be $10^{-13} \mu_{\mathrm{B}}$, the density is $10^{10} \mathrm{~g} \mathrm{~cm}^{-3}$, and the gradient of the electron fraction is $\mathrm{d} Y_{e} / \mathrm{d} r \simeq 10^{-7}$ $\mathrm{cm}^{-1}$.

\section{Time evolution of the neutrino flux}

The process of helicity flip for a Dirac neutrino with a magnetic moment, which can lead to interesting observational consequences when the expected neutrino signal from an imminent supernova explosion is studied in detail, can be realized. According to existing views, during the explosion of a Galactic supernova at a distance up to $10 \mathrm{kpc}$, the expected number of neutrino events in the Super-Kamiokande detector will be $\sim 10^{4}$. This will allow the time evolution of the neutrino flux to be recorded with a good accuracy.

In the presence of a sufficiently strong magnetic field in the supernova envelope, not only the above-mentioned conversion of right-handed neutrinos into left-handed ones, $\nu_{R} \rightarrow \nu_{L}$ [22, 23, but also the conversion of active electron neutrinos and antineutrinos of the main neutrino flux into a form sterile with respect to weak interactions, $\nu_{L} \rightarrow \nu_{R}, \bar{\nu}_{R} \rightarrow \bar{\nu}_{L}$, is possible.

Numerical analysis of Eq. (41) shows that after its passage through the resonance region $\left(Y_{e}=1 / 3\right)$, the flux of left-handed neutrinos is attenuated as a result of the above conversion by the factor $W_{L L}$, which has the meaning of the survival probability of left-handed neutrinos, $\nu_{e L} \rightarrow \nu_{e L}$, or, in other words, the transparency. Figure 1 shows the characteristic variation in $W_{L L}$ when passing through the resonance point (placed here at the coordinate origin) for various magnetic field strengths. We see that the supernova envelope in the presence of a sufficiently strong magnetic field is virtually opaque to active electron neutrinos and antineutrinos, which can cause the expected neutrino signal from the supernova to be attenuated.

A more detailed analysis of the numerical solution of Eq. (44) allows us to establish a relationship between the magnetic field strength and parameters of the medium in the supernova envelope, on the one hand, and the survival probability of active neutrinos $W_{L L}$, on the other 
hand. Using typical scales of parameters in the region under consideration, see [32, 33],

$$
\frac{\mathrm{d} Y_{e}}{\mathrm{~d} r} \sim 10^{-7} \mathrm{~cm}^{-1}, \quad \rho \sim 10^{10} \mathrm{~g} \mathrm{~cm}^{-3},
$$

we find an approximation formula,

$$
\begin{aligned}
& \frac{B_{\perp}(t)}{B_{e}}=f\left(W_{L L}\right)\left(\frac{10^{-13} \mu_{\mathrm{B}}}{\mu_{\nu}}\right) \times \\
& \times\left(\frac{\rho(t)}{10^{10} \mathrm{~g} \mathrm{~cm}^{-3}}\right)^{1 / 2}\left(\frac{\mathrm{d} Y_{e}}{\mathrm{~d} r}(t) \times 10^{7} \mathrm{~cm}\right)^{1 / 2} .
\end{aligned}
$$

Here, the factor

$$
f\left(W_{L L}\right)=0.88 \frac{\left(1-W_{L L}\right)^{0.62}}{\left(W_{L L}\right)^{0.13}}
$$

characterizes the degree of adiabaticity of the conversion process. The literal adiabaticity corresponds to the limit $f \rightarrow \infty$, when $W_{L L} \rightarrow 0$; in this case, the left-handed neutrinos are completely converted into right-handed ones, $W_{L R}=\left(1-W_{L L}\right) \rightarrow 1$.

The conservative value of $10^{-13} \mu_{\mathrm{B}}$ introduced in Eq. (9) as the scale for the neutrino magnetic moment was chosen to be an order of magnitude smaller than limit (3), so that the conversion of sterile neutrinos produced in the supernova core through the first of the above mechanisms into active ones did not distort the supernova explosion dynamics. Thus, we can use the parameters of the explosion model without allowance for the influence of the neutrino magnetic moment. Our analysis based on detailed data on the radial distributions and time evolution of physical properties in a supernova core obtained in the specific model of a successful explosion [34] showed that the gradient of the electron fraction $\mathrm{d} Y_{e} / \mathrm{d} r$ in Eq. (9) grows fairly rapidly with time at point $Y_{e}=1 / 3$ and, thus, the envelope becomes more transparent to active neutrinos at a fixed magnetic field strength. This means that the neutrino signal from the supernova can be attenuated within some limited time interval after its explosion. Thus, if the Dirac neutrino had a magnetic moment and if the magnetic field in the supernova envelope were sufficiently strong, then the characteristic effect of a significant attenuation of the initial neutrino signal intensity peak predicted by supernova models could take place. For example, there would be a tenfold reduction in the neutrino signal $\left(W_{L L}=0.1\right)$ for typical parameters of the medium at a magnetic field strength

$$
\begin{aligned}
& B_{\perp}=4.9 \times 10^{13}\left(\frac{10^{-13} \mu_{\mathrm{B}}}{\mu_{\nu}}\right) \times \\
& \times\left(\frac{\rho}{10^{10} \mathrm{~g} \mathrm{~cm}^{-3}}\right)^{1 / 2}\left(\frac{\mathrm{d} Y_{e}}{\mathrm{~d} r} \times 10^{7} \mathrm{~cm}\right)^{1 / 2} .
\end{aligned}
$$

Note that the possible strengths of a magnetic field generated in a supernova envelope are believed to reach $10^{16} \mathrm{G}[35-40]$.

\section{The neutrino signal from SN 1987A}

It is of interest to compare observational predictions of the effect being discussed with the only (to date) neutrino signal from SN 1987A, when three underground neutrino detectors, Kamiokande-II [11,12, IMB [13, 14], and the Baksan scintillation telescope [15], recorded electron antineutrinos in the reaction $\bar{\nu}_{e}+p \rightarrow n+e^{+}$for the first time. The neutrino signal recorded by the LSD detector [16] $4 \mathrm{~h} 43 \mathrm{~min}$ earlier than these three detectors requires a separate analysis and we disregard it.

It should be recognized that the statistics of neutrino events from SN 1987A is, of course, insufficient for firm conclusions about the time evolution of the neutrino flux to be reached: 
the Kamiokande, IMB, and Baksan detectors recorded eleven (one more event was attributed to the background), eight, and five events (one more event was attributed to the background), respectively. Combining the data from all three detectors to study the time evolution of the neutrino signal is a serious problem. First, the signal timing accuracy was different: it was \pm $50 \mathrm{~ms}$ for IMB, for $\pm 1 \mathrm{~min}$ for Kamiokande, and $\pm 2 \mathrm{~s}$ for the Baksan telescope. Second, the detectors had different sensitivity thresholds: at an energy of positrons below $27 \mathrm{MeV}$, the efficiency of their detection by the IMB detector dropped below $30 \%$; for the Kamiokande and Baksan detectors, the corresponding thresholds were 7 and $9 \mathrm{MeV}$.

The simplest and, at the same time, not unfounded solution of the signal timing problem is that the first events in the series from different detectors are declared to be coincident in time and an appropriate time shift is made (see, e.g., [41]). The reason for this solution is that, according to the existing supernova explosion models (see, e.g., [32,33]), the supernova core luminosity through electron antineutrinos reaches its maximum within about $30 \mathrm{~ms}$ after the bounce and then decreases fairly rapidly after $200 \mathrm{~ms}$. Thus, the probability of detecting the antineutrinos produced precisely in this time interval is maximal. Making the first events coincident in time in all three detectors and choosing this instant as the zero time $t=0$, we obtain a set of 24 events [12,14,15] in the time interval from 0 to $12.4 \mathrm{~s}$, with 17 events occurring during the first $3 \mathrm{~s}$. However, the time distribution of these events exhibits no initial peak. Thus, it may well be that the time evolution of the only observed neutrino signal from SN 1987A confirms the attenuation of the initial peak described above.

\section{$5 \quad$ Neutrino pulsar}

Note another possible interesting manifestation of the neutrino magnetic moment. If a magnetar with a poloidal magnetic field of $10^{14}-10^{15} \mathrm{G}$ is formed during a supernova explosion, then, given that Eqs. (4) and (9) contain the transverse magnetic field component $B_{\perp}$, the neutrinos can avoid the conversion of their helicity only in a narrow region near the poles. When the nascent magnetar rotates around an axis that does not coincide with its magnetic moment and if we are lucky with the orientation of the rotation axis, the neutrino signal will have a pulsating behavior, as is illustrated in Fig. 2, i.e., a kind of a neutrino pulsar can be observed.

It should be noted that, strictly speaking, the described influence of a strong magnetic field when the neutrino has a magnetic moment on the time evolution of the neutrino signal is incomplete without allowance for the effects of neutrino flavor oscillations (see, e.g., [42]). The combined action of these effects on the neutrino flux requires a special study.

\section{Discussion}

We showed that if the Dirac neutrino had a magnetic moment and if the magnetic field in the supernova envelope were sufficiently strong, then the characteristic effect of a significant attenuation of the initial neutrino signal intensity peak predicted by supernova models could take place. For instance, at typical parameters of the medium and at a neutrino magnetic moment of $\sim 10^{-13} \mu_{\mathrm{B}}$, i.e., an order of magnitude smaller than the existing astrophysical limit, there would be a tenfold reduction in the neutrino signal even at a magnetic field strength of the order of the critical one $B_{e}$.

Remarkably, as our analysis showed, the time evolution of the only observed neutrino signal from SN 1987A may confirm this attenuation of the initial neutrino peak.

If a magnetar with a poloidal magnetic field is formed in a supernova explosion, then the neutrino signal will have a pulsating behavior, i.e., a kind of a neutrino pulsar can be observed, when it rotates around an axis that does not coincide with its magnetic moment and when the orientation of its rotation axis is "lucky". 


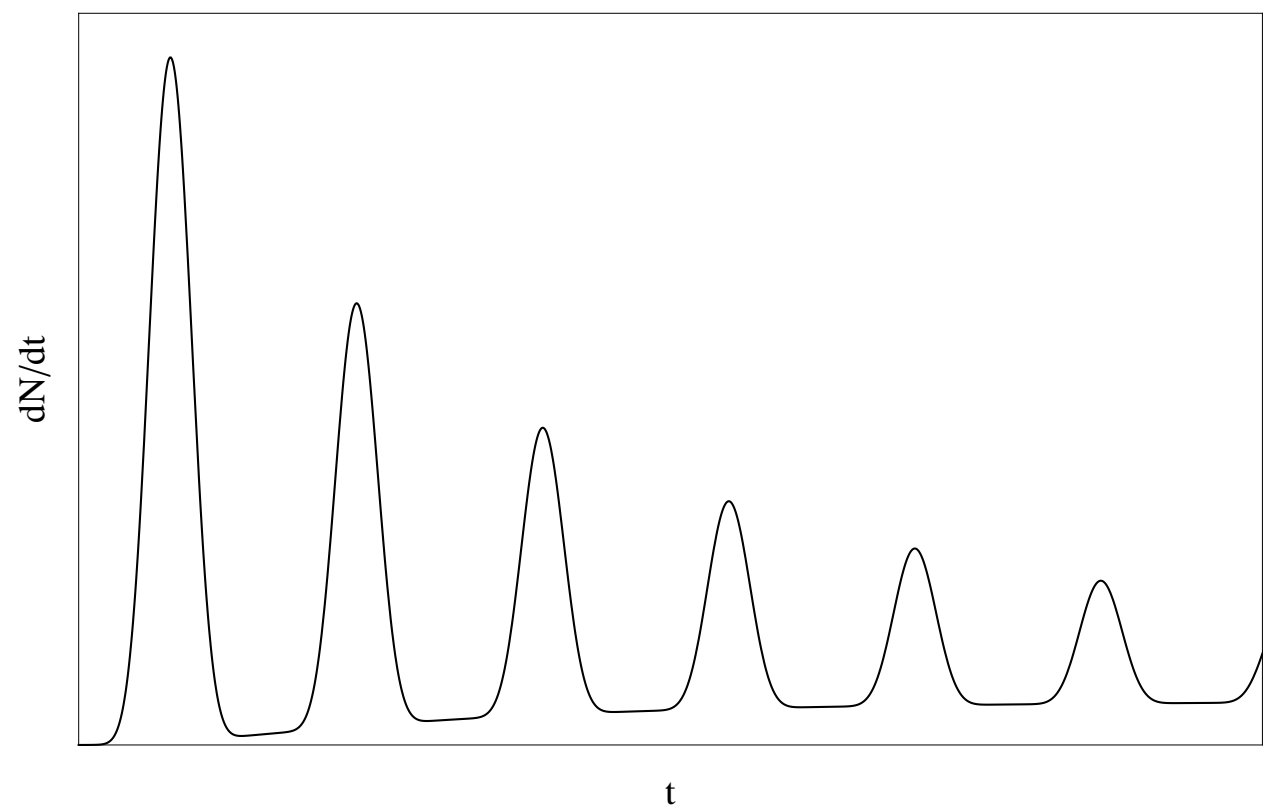

Figure 2: Illustration of the pulsating behavior of the neutrino signal from a nascent magnetar rotating around an axis that does not coincide with its magnetic moment, a neutrino pulsar.

\section{Acknowledgements}

A.K. and N.M. express their deep gratitude to the organizers of the Seminar "Quarks-2010" for warm hospitality. We are grateful to Hans-Thomas Janka, Lorenz Hüdepohl and Bernhard Müller for providing us with detailed data on radial distributions and time evolution of physical parameters in the supernova core, obtained in their model of supernova explosion and protoneutron star cooling. We thank A.A. Gvozdev and I.S. Ognev for useful remarks.

This study was carried out within the framework of the "Scientific and Scientific-Educational Personnel of Innovational Russia" Federal Goal-Oriented Program for 2009-2013 (State contract no. P2323) under partial financial support by the Ministry of Education and Science of the Russian Federation according to the "Development of Scientific Potential of Higher School" Program (project no. 2.1.1/510).

\section{References}

[1] B. W. Lee and R. E. Shrock, Phys. Rev. D 16, 1444 (1977).

[2] K. Fujikawa and R.E. Shrock, Phys. Rev. Lett. 45, 963 (1980).

[3] E. M. Lipmanov, Yad. Fiz. 6, 541 (1967) [Sov. J. Nucl. Phys. 6, 395 (1967)].

[4] E. M. Lipmanov and N. V. Mikheev, Pis'ma Zh. Eksp. Teor. Fiz. 7, 139 (1968) [JETP Lett. 7, 107 (1968)].

[5] E. M. Lipmanov, Zh. Eksp. Teor. Fiz. 55, 2245 (1968) [Sov. Phys. JETP 28, 1191 (1968)].

[6] J. C. Pati and A. Salam, Phys. Rev. D 10, 275 (1974).

[7] M. A. B. Bég, R. Budny, R. N. Mohapatra and A. Sirlin, Phys. Rev. Lett. 38, 1252 (1977).

[8] J.E. Kim, Phys. Rev. D 14, 3000 (1976). 
[9] W. J. Marciano and A. I. Sanda, Phys. Lett. B 67, 303 (1977).

[10] M. A. B. Bég, W. J. Marciano and M. Ruderman, Phys. Rev. D 17, 1395 (1978).

[11] K. Hirata, T. Kajita, M. Koshiba et al. (Kamiokande-II Collab.), Phys. Rev. Lett. 58, 1490 (1987).

[12] K. Hirata, T. Kajita, M. Koshiba et al. (Kamiokande-II Collab.), Phys. Rev. D 38, 448 (1988).

[13] R. M. Bionta, G. Blewitt, C. B. Bratton et al. (IMB Collab.), Phys. Rev. Lett. 58, 1494 (1987).

[14] C. B. Bratton, D. Casper, A. Ciocio et al. (IMB Collab.), Phys. Rev. D 37, 3361 (1988).

[15] E. N. Alexeyev, L. N. Alexeyeva, V. I. Volchenko and I. V. Krivosheina, Pis'ma Zh. Eksp. Teor. Fiz. 45, 461 (1987) [JETP Lett. 45, 589 (1987)].

[16] M. Aglietta, G. Badino, G. Bologna et al. (LSD Collab.), Europhys. Lett. 3, 1315 (1987).

[17] R. Barbieri and R. N. Mohapatra, Phys. Rev. Lett. 61, 27 (1988).

[18] A. Ayala, J. C. D’Olivo and M. Torres, Phys. Rev. D 59, 111901 (1999).

[19] A. Ayala, J. C. D’Olivo and M. Torres, Nucl. Phys. B 564, 204 (2000).

[20] A. V. Kuznetsov and N. V. Mikheev, Journ. Cosmol. Astropart. Phys. 11, 031 (2007).

[21] A. V. Kuznetsov, N. V. Mikheev and A. A. Okrugin, Int. Journ. Mod. Phys. A 24, 5977 (2009).

[22] A. Dar, Neutrino magnetic moment may solve the supernovae problem, Inst. Advanced Study Preprint-87-0178, Princeton, 1987.

[23] M. B. Voloshin, Phys. Lett. B 209, 360 (1988).

[24] A. V. Kuznetsov, N. V. Mikheev and A. A. Okrugin, Pis'ma Zh. Eksp. Teor. Fiz. 89, 115 (2009) [JETP Lett. 89, 97 (2009)].

[25] R. A. Anikin, A. V. Kuznetsov and N. V. Mikheev, Pis'ma Astron. Zh. 36, 714 (2010) [Astron. Lett. 36, 680 (2010)].

[26] M. B. Voloshin, M. I. Vysotskii and L. B. Okun', Yad. Fiz. 44, 677 (1986) [Sov. J. Nucl. Phys. 44, 440 (1986)].

[27] M. B. Voloshin and M. I. Vysotskii, Yad. Fiz. 44, 845 (1986) [Sov. J. Nucl. Phys. 44, 544 (1986)].

[28] L. B. Okun', Yad. Fiz. 44, 847 (1986) [Sov. J. Nucl. Phys. 44, 546 (1986)].

[29] M. B. Voloshin, M. I. Vysotskii and L. B. Okun', Zh. Eksp. Teor. Fiz. 91, 754 (1986) [Sov. Phys. JETP 64, 446 (1986)]; Erratum: ibid. 92, 368 (1987) [Sov. Phys. JETP 65, 209 (1987)].

[30] L. B. Okun', Yad. Fiz. 48, 1519 (1988) [Sov. J. Nucl. Phys. 48, 967 (1988)].

[31] S. I. Blinnikov and L. B. Okun', Pis'ma Astron. Zh. 14, 867 (1988) [Sov. Astron. Lett. 14, 368 (1988)].

[32] R. Buras, M. Rampp, H.-Th. Janka and K. Kifonidis, Astron. Astrophys. 447, 1049 (2006). 
[33] H. A. Bethe, Rev. Mod. Phys. 62, 801 (1990).

[34] L. Hüdepohl, H.-Th. Janka and B. Müller, unpublished results (private communication).

[35] G. S. Bisnovatyi-Kogan, Astron. Zh. 47, 813 (1970) [Sov. Astron. 14, 652 (1970)].

[36] G. S. Bisnovatyi-Kogan, Physical Questions of Stellar Evolution (Nauka, Moscow, 1989) [in Russian].

[37] P. Bocquet, S. Bonazzola, E. Gourgoulhon and J. Novak, Astron. Astrophys. 301, 757 (1995).

[38] H. C. Spruit, Astron. Astrophys. 341, L1 (1999).

[39] C. Y. Cardall, M. Prakash and J. M. Lattimer, Astrophys. J. 554, 322 (2001).

[40] N. V. Ardeljan, G. S. Bisnovatyi-Kogan and S. G. Moiseenko, Mon. Not. Roy. Astron. Soc. 359, 333 (2005).

[41] E. N. Alexeyev, L. N. Alexeyeva, I. V. Krivosheina and V. I. Volchenko, Phys. Lett. B 205, 209 (1988).

[42] J.P. Kneller, G. C. McLaughlin and J. Brockman, Phys. Rev. D 77, 045023 (2008). 